\title{
Weniger Erkrankte und mehr Über- lebende dank Darmkrebsvorsorge
}

\author{
Urs Marbet \\ Prof. Dr. med., senior consultant Kantonsspital Uri, Mitglied FMH
}

\begin{abstract}
Darmkrebs gehört zu den häufigsten Krebsarten in der Schweiz, die zum Tode führen. Chemotherapien, Bestrahlung, Operationen, künstlicher Darmausgang verschlechtern die Lebensqualität massiv und verursachen hohe Kosten. Die Darmkrebsvorsorge verhindert viele Erkrankungen und erhöht durch frühe Diagnostik Lebenszeit und -qualität. Voraussetzung ist eine exzellente Qualität vor allem der Kolonoskopie.
\end{abstract}

\section{Kurz-Zusammenfassung}

Wird Darmkrebs erst aufgrund von Beschwerden gefunden, ist er oft bereits in einem fortgeschrittenen Stadium und die Überlebenschancen reduziert. Die Behandlung ist dann mit Operationen, Chemotherapien und Bestrahlung für die Patienten sehr belastend und zudem sehr kostenintensiv.

Darmspiegelungen im Rahmen von Vorsorgeuntersuchungen können Darmkrebs mehrheitlich im Frühstadium entdecken und die Heilungschancen verbessern. Nicht selten ist sogar eine sofortige endoskopische Entfernung möglich. Zudem verhindert die Abtragung der Vorstufen oft sogar die Entstehung von Krebs und macht zusätzliche Behandlungen überflüssig. Voraussetzung einer solchen effektiven und kostensparenden Vorsorge ist eine hochstehende Qualität der Kolonoskopie: Eine hohe Entdeckungsrate von Krebsvorstufen setzt exzellente Instrumente und eine extrem sorgfältige Untersuchung des Darms bei genügend langsamer Rückzugzeit voraus. Diese Qualitätsstandards gilt es auch gegen Sparbemühungen zu verteidigen, da verpasste Adenome und eine Zunahme an Darmkrebs die Patienten, aber auch alle andere Versicherten teurer zu stehen kämen.

\section{Was nützt die Kolonoskopie dem Patienten?}

Herr ZA, 69-jährig, beschwerdefrei, ohne familiäre Belastung, kam kürzlich zur vorsorglichen Darmspiegelung. Dabei wurde ein mehrere Zentimeter grosses Geschwulst, ein Adenom, kurz vor dem After und mehrere Adenome im übrigen Dickdarm gefunden und bei der Spiegelung entfernt. Wäre Herr ZA erst beim Auftreten von Beschwerden gekommen, wie dies vor wenigen Jahren nicht anders möglich war, hätte er operiert werden müssen, hätte einen künstlichen Darmausgang bekommen und wäre mit grosser Wahrscheinlichkeit später am Darmkrebs gestorben.

\section{Darmkrebsvorsorge verbessert die Lebens- chancen bei einer häufigen Krankheit}

Jedes Jahr erkranken etwa 4100 Menschen in der Schweiz an Dickdarmkrebs und etwa 1600 sterben daran [1]. Betrachtet man die durch Krebserkrankungen bedingten verlorenen Lebensjahre, steht der Darmkrebs unter den Verursachern an dritter Stelle [1]. Da die Erkrankungsraten mit dem Alter steigen, kommt dem Darmkrebs und seiner Prävention in unserer alternden Gesellschaft wachsende Bedeutung zu.

Seit gut 15 Jahren werden in Uri sämtliche Leute registriert, bei denen ein Darmkrebs diagnostiziert wird. Dabei wird unterschieden, ob die Tumoren im Rahmen einer Darmkrebsvorsorge entdeckt wurden oder ob die Leute wegen Beschwerden zur Kolonoskopie kamen. Die Daten zeigen ein klares Bild: Bei den Personen mit Beschwerden haben leider mehr als $80 \%$ das gut behandelbare Frühstadium bereits überschritten, die meisten haben schon Absiedler in Lymphknoten und oft bereits Metastasen in andern Organen [2, 3]. Bei den beschwerdefreien Personen hingegen wird Darmkrebs bei 70\% bereits in einem Stadium I, also der frühsten Form mit sehr guten Heilungschancen, gefunden. Während von den Darmkrebspatienten, deren Tumor nicht im Rahmen der Vorsorge gefunden wurde, gegen $40 \%$ später am Tumor sterben, sind dies unter den Darmkrebspatienten aus den Vorsorgeuntersuchungen keine 10\%.

\section{Darmkrebsvorsorge verhindert Erkran- kungen - und spart Behandlungen}

Die Behandlung von Darmkrebs ist belastend und teuer. Dank Screening wird Darmkrebs aber immer seltener: 
Weil bei der Kolonoskopie zahlreiche Adenome, also Krebsvorstufen, entfernt werden, kann Darmkrebs oft verhütet werden. Unsere Daten zeigen, dass mittels vorsorglicher Darmspiegelung innert zehn Jahren bei 1000 Leuten im Alter über 50 Jahren 8 bis 10 Karzinome verhindert werden können [3]. In den USA, wo die Darmkrebsvorsorge seit Jahren intensiv durchgeführt wird, hat sich die Zahl neuauftretender Darmkrebserkrankungen bei den über 55 Jährigen etwa halbiert [4]. Auch in der Schweiz, wo die Darmkrebsvorsorge seit 2013 kassenpflichtig ist, ist diese Tendenz zumindest im distalen Dickdarm erkennbar [5].

Darmkrebs im Stadium I, also dem Tumorstadium, das bei der Vorsorge mehrheitlich angetroffen wird, kann heute nicht selten endoskopisch gleich entfernt werden, was früher undenkbar war. Eine Bestrahlung oder Chemotherapie bleibt diesen Patienten dadurch erspart. Zwar leben auch Leute mit einem fortgeschrittenen Darmkrebs heute weit länger als noch vor einigen Jahren, müssen aber Chemotherapien, Bestrahlungen und operative Eingriffe zum Entfernen von Metastasen meist aus Leber und Lunge über sich ergehen lassen. Dies schlägt sich zwangsläufig auch auf die Gesundheitskosten nieder: Die Behandlung eines Darmkrebs, der erst beim Auftreten von Beschwerden gefunden wird, dürfte heute im Schnitt 100000 Franken kosten, Tendenz dank noch besserer, aber auch teurerer Therapien steigend. Deshalb heisst die Frage heute nicht mehr, ob die Darmkrebsvorsorge kosteneffizient ist, sondern zunehmend, wie viele Kosten sie einsparen könnte. Dieses Potential hängt auch von der Akzeptanz und Adhärenz der Leute ab. Da beispielsweise Stuhltests besser akzeptiert werden als Darmspiegelungen, dürften vor allem Vorsorgeprogramme sparen helfen, die bei Leuten mit niedrigem Tumorrisiko auch einen effizienten modernen Stuhltest anbieten.

\section{Voraussetzung effektiver Vorsorge: hohe Qualität}

Die wichtigste Voraussetzung, damit eine Kolonoskopie im Rahmen der Darmkrebsvorsorge effektiv die Entstehung von Tumoren verhindern und die Überlebenschancen verbessern kann, ist eine hohe Qualität. Die Qualität einer Kolonoskopie umfasst die perfekte Vorbereitung des Darmes vor der Spiegelung, die kompetente Untersuchung durch gut ausgebildete Endoskopiker mit exzellenten High-Tech-Instrumenten, die gleichzeitige vollständige Entfernung der Vorstufen, eine optimale Nachkontrolle von Patienten mit Be- funden oder relevanter familiärer Tumorbelastung sowie eine hohe Teilnahmerate bei Darmspiegelung und Stuhltestprogrammen. Es konnte mehrfach gezeigt werden, dass eine gute Qualität mit einer hohen Entdeckungsrate von Adenomen einhergeht. Vor allem im proximalen Dickdarm können Tumoren und ihre heimtückischen flachen Vorstufen (flache serratierte Adenome) auch bei der Darmspiegelung leicht übersehen werden.

Angesichts der mit Hilfe qualitativ hochstehender Kolonoskopien erhöhten Überlebensraten, der reduzierten Belastungen für Patienten und auch angesichts der damit eingesparten Kosten sollte uns diese Qualität etwas wert sein. Neben den dafür notwendigen, im Einkauf und im Unterhalt teuren, aber exzellenten Instrumenten braucht es eine extrem sorgfältige geduldige Untersuchung, damit die versteckten, kaum erkennbaren Adenome gefunden werden können. Riesige Untersuchungen weltweit zeigten, dass Darmkrebs nach der Spiegelung desto seltener auftritt, je mehr Adenome bei der Kolonoskopie gefunden werden. Mehrere Studien zeigten zudem, dass diese Sorgfalt mit der Zeit korreliert, die sich der Untersucher für die Darmspiegelung nimmt. Die für die Untersuchung aufgewendete Zeit steht also in direktem Zusammenhang mit ihrem Nutzen. Deshalb wurde eine minimale Zeit definiert und die Messung der sogenannten Rückzugzeit des Instrumentes bei jeder Untersuchung etabliert. Diese Standards gilt es zu wahren und auch gegen Sparvorgaben zu verteidigen, denn eine unrealistische Begrenzung der für Darmspiegelungen aufzuwendenden Zeit provoziert zwangsläufig raschere und damit unsorgfältigere Untersuchungen und gefährdet den Nutzen der Darmkrebsvorsorge: Sollten verpasste Adenome und Tumore wieder zu mehr Darmkrebs und fortgeschritteneren Tumorstadien führen, würde dies nicht nur die Patienten und ihr Umfeld, sondern auch alle Versicherten unter dem Strich viel kosten.

Referenzen

1 Bundesamt für Statistik (Hrsg.). Schweizerischer Krebsbericht 2015. Stand und Entwicklungen. Neuchâtel, 2016.

2 Marbet UA, Bauerfeind P, Brunner J, Dorta G, Valloton JJ, Delco F: Colonoscopy ist the preferred colorectal cancer screening method in a population-based programm. Endoscopy 2008;40:650-5.

3 Manser CN, Bachmann LM, Brunner J, Hunold F, Bauerfeind P, Marbet UA: Colonoscopy screening markedly reduces the occurrence of colon carcinomas and carcinoma related death a closed cohort study. Gastrointest Endosc 2012;76:110-7.

4 Siegel RL, Fedewa SA. Anderson WF, Miller KD, Ma J, Rosenberg PS, Jemal A: Colorectal cancer incidence patterns in the United States, 1974-2013. J Natl Cancer Inst. 2017;109:djw322 JNCI 2017.

5 Lorez M, Marbet U, Arndt V: subsite-specific colorectal cancer trends in Switzerland (1989-2012). Schweiz Krebsbulletin 2016;1:67-74 